\title{
PERFORMANCE EVALUATION METHOD CONSIDERING SHOCK WAVE IN NOZZLE FOR HIGH-VOLTAGE CIRCUIT BREAKER
}

\author{
M. Terada ${ }^{a, c, *}$, H. Urai ${ }^{a}$, T. Nishimura ${ }^{b}$, Y. YoKomizU ${ }^{c}$ \\ ${ }^{a}$ Research 83 Development Group, Hitachi, Ltd., 7-1-1, Oomika-cho, Hitachi-shi, Ibaraki-ken, 319-1292, Japan \\ ${ }^{b}$ Energy Business Unit, Hitachi, Ltd., 1-1-1, Kokubu-cho, Hitachi-shi, Ibaraki-ken, 316-8501, Japan \\ ${ }^{c}$ Department of Electrical Engineering, Nagoya University, Chikusa-ku, Furo-cho, Nagoya-shi, Aichi-ken, \\ 464-8603, Japan \\ * masanao.terada.br@hitachi.com
}

\begin{abstract}
Interrupting-performance indexes that reflect the physical properties of gas flow are proposed for a breaker terminal fault of a high-voltage circuit breaker. A method of evaluating the shock wave and flow velocity was developed that uses a theoretical equation of supersonic flow which takes into account the nozzle's shape and stagnation pressure. The predicted flow velocities and positions at which the shock wave forms have strong correlations with the test results.
\end{abstract}

Keywords: supersonic flow, shock wave, flow velocity, circuit breaker.

\section{Introduction}

High-voltage circuit breaker was required to have a low operating energy for the sake of reliability and economy. Self-blast circuit breakers are widely used as they reduce the operating energy. They utilize a pressure build-up with ablation of a polytetrafluoroethylene (PTFE) insulation nozzle by arc energy, resulting in a minimization of the reaction force of mechanical compression.

Breaker terminal fault (BTF) test duties have a wide range of interrupting current. The self-blast circuit breaker utilizes ablation of the PTFE insulation nozzle by arc energy. Accordingly, the ratio of pressure rise to current is higher than that of conventional puffertype circuit breakers. Therefore, at low currents, it is important that a small pressure rise in a pressure generating process is effectively converted into a flow generating process around zero current and hot gas is exhausted. An interrupter can be designed around a complex gas flow varying in time and space by using a computational fluid dynamics simulation technique taking into account the effects of arc heating and ablation [1-3]. Furthermore, the dielectric strength for transient recovery voltage (TRV) briefly after current interruption in a BTF can be evaluated by combining the flow simulation with an electric-field simulation $[4,5]$. The dielectric strength is determined by the local density of the gas and the local electrical field. The dielectric strength is high enough if the space between electrodes is filled with a low-temperature, high-density gas. On the other hand, if the pressure is not high enough, there will be high-temperature and low-density spots between the electrodes, and in that case, the dielectric strength should be evaluated with a simulation technique.

Such a design requires a lot of trials with different structural parameters and exhaustive considerations.
In this case, macroscopic evaluations focused on flow conditions will be effective. References $[6,7]$ evaluate the interrupting performance in correlation with the flow properties. The current study deals with supersonic flow inside the nozzle at the time around a peak value of the TRV briefly after current interruption, where performance indexes evaluating the dielectric strength are the flow velocity and shock wave position. As mentioned above, to increase the dielectric strength, the density in the nozzle should be high. One way of increasing the density is to decrease the temperature in the area between the electrodes. To do so effectively, it is important that the flow be smooth to promote the exhaust of hot gas. Thus, a stable and strong flow should be generated between the electrodes. Moreover, it is important for the shock wave to be generated on the downstream side so as to make the gas flow stable and to increase the flow velocity so as to make it powerfully flowing.

The purpose of this work is to introduce performance indexes evaluating the dielectric strength in the form of the flow velocity and the shock wave position for theoretically evaluating the physical properties of supersonic flows in nozzles in reference with the experimental data of whether the dielectric breakdown occurs or not with actual interrupting tests.

\section{Flow conditions in region between electrodes}

In the interrupting process, a supersonic flow forms in the divergence area of the nozzle and a shock wave is generated downstream of it. The gas flow is accelerated upstream of the shock wave and stalls downstream of the shock wave. Therefore, it is considered that there are two indexes related to dielectric strength briefly after current interruption. The first is the position of the shock wave. In particular, the shock wave 
should not form in front of the pin electrode. The second one is the maximum flow velocity in the region upstream of the shock wave.

Figure 1 shows the above ideas. Figure 1 (a) shows the case of a shock wave generated in front of the pin electrodes. In this case, the flow velocity at the tip of the pin electrode is small, resulting in hot gas remaining due to flow retention. Figure 1 (b) shows a shock wave formed to the rear of the pin electrodes. The drawing superimposes the shock wave at the front of the pin electrode, which acts as a barrier. In this case, the flow around the pin electrode is supersonic. In addition, the dielectric strength at the tip of the pin electrode is high because the area around that point in which hot gas is retained is narrow and at low temperature. Therefore, it is considered that the flow condition of Figure 1 (b) is suitable for increasing dielectric strength.

To generate a shock wave downstream of the nozzle divergence area, the pressure at the stagnation point should be increased. One way of increasing the pressure is to increase the amount of nozzle ablation and to reduce the flow resistance through the pressure build-up chamber. Another way is to narrow the nozzle divergence angle. However, the nozzle divergence angle should be enlarged to increase the flow velocity. The design of the nozzle should thus consider this trade-off relationship.

It is important for the evaluation to include the effect of varying the gas density and the temperature in combination with the electric field upstream and/or downstream of the shock wave. Figure 2 shows temperature distribution at $100 \mu \mathrm{s}$ after current zero with CFD simulation in ANSYS Fluent which is based on the finite-volume method applied with the discrete ordinates radiation model and the realizable k- $\epsilon$ turbulence model. The nozzle ablation due to radiation heat transfer from the arc is also considered. The dielectric strength of this simulated structure is relatively low. The flow condition of the simulation result is close to the condition of the Figure 1 (a), that is, the shock wave is generated in front of the pin electrode resulting in hot gas remaining due to flow retention. One approach to design the high-voltage circuit breaker is to find the structure eliminating local weak points using with CFD simulation. Another approach is to determine the policy of the performance improvement evaluating the macroscopic performance-indexes since the interrupting process has a transient phenomena and a large variation. The target of this study is the latter approach.

\section{Calculation method}

The position of the shock wave and the flow velocity were calculated using the theoretical equation of supersonic flow. To evaluate the flow properties in terms of the pressure and the shape of the nozzle, it is considered that the pin electrode does not exist.

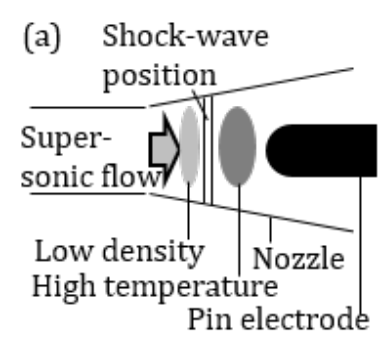

(b)

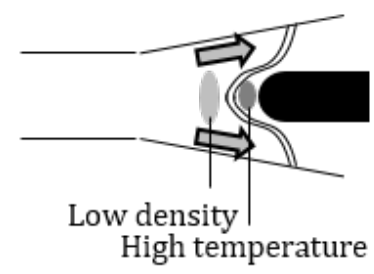

Figure 1. Shock wave position. (a) shows a shock wave generated in front of the pin electrodes. (b) shows a shock wave generated to the rear of the pin electrodes.

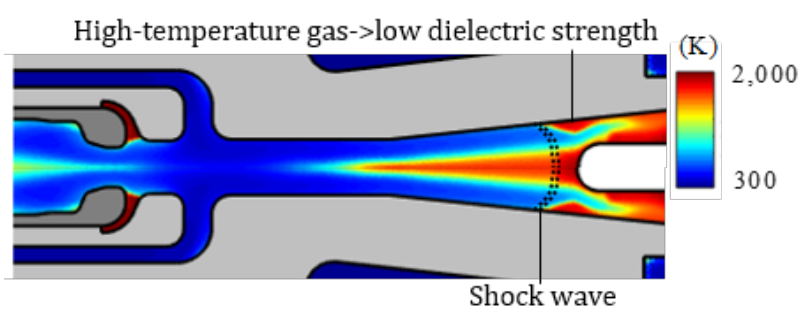

Figure 2. Temperature distribution at $100 \mu \mathrm{s}$ after current zero with CFD simulation.

Figure 3 shows the calculation model for simulating supersonic flow.

The method of calculating the position of the shock wave is described below. Equations (1)-(3) show how the cross-sectional area ratio and Mach number are related to the stagnation pressure [8].

$$
\begin{gathered}
\frac{A_{\mathrm{e}}}{A^{*}}=\frac{\left(\frac{2}{\gamma+1}\right)^{\frac{1}{\gamma-1}}\left(\frac{P_{0}}{P_{\mathrm{e}}}\right)^{\frac{1}{\gamma}}}{\sqrt{\left(\frac{\gamma+1}{\gamma-1}\right)\left\{1-\left(\frac{P_{0}}{P_{\mathrm{e}}}\right)^{\frac{1-\gamma}{\gamma}}\right\}}} \\
M_{\mathrm{e}}^{2}=\frac{2}{\gamma-1}\left\{\left(\frac{P_{0}}{P_{\mathrm{e}}}\right)^{\frac{\gamma-1}{\gamma}}-1\right\} \\
P_{\mathrm{h}}=P_{0} \frac{2 \gamma M_{\mathrm{e}}^{2}-(\gamma-1)}{\gamma+1}\left(1+\frac{\gamma-1}{2} M_{\mathrm{e}}^{2}\right)^{-\frac{\gamma}{\gamma-1}}
\end{gathered}
$$

where $P_{0}, A^{*}, A_{\mathrm{e}}, P_{\mathrm{e}}, M_{\mathrm{e}}, P_{\mathrm{h}}$, and $\gamma$ are the stagnation pressure, cross-sectional area of the nozzle throat, cross-sectional area of the nozzle at the shock wave's position, pressure upstream of the shock wave, Mach number upstream of the shock wave, pressure downstream of the shock wave, and ratio of specific heats, respectively. The above equations are used to calculate $A_{\mathrm{e}}$ at the shock wave, where $P_{\mathrm{h}}$ equals $P_{\mathrm{b}}$ witch is the back-pressure. The distance from the nozzle throat exit $L_{\mathrm{s}}$ to the position of the shock wave is expressed as

$$
L_{\mathrm{s}}=\frac{\sqrt{\frac{A_{\mathrm{e}}}{\pi}}-\sqrt{\frac{A^{*}}{\pi}}}{\tan \theta}
$$

where $\theta$ is the nozzle divergence angle. 


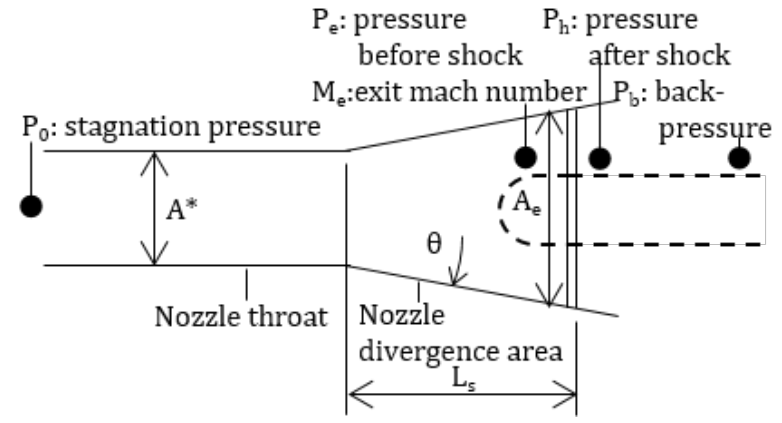

Figure 3. Calculation model for supersonic flow.

\begin{tabular}{ccc}
\hline \hline No. & Th. len. (a.u.) & Div. angle (a.u.) \\
\hline Case A & 1.0 & 1.0 \\
Case B & 0.9 & 1.0 \\
Case C & 1.0 & 1.2 \\
Case D & 0.9 & 0.9 \\
\hline \hline
\end{tabular}

Table 1. Configurations of test structure.

Second, the flow velocity $u_{\mathrm{e}}$ under the back-pressure condition where the shock wave at the curved end of the tip of the pin is generated is expressed as

$$
u_{\mathrm{e}}=M_{\mathrm{e}} c_{\mathrm{e}}=M_{\mathrm{e}} \sqrt{\gamma R T_{\mathrm{e}}}
$$

where $M_{\mathrm{e}}, c_{\mathrm{e}}, T_{\mathrm{e}}$, and $R$ are the Mach number upstream of the shock wave, the speed of sound, temperature at the shock wave's position, and the gas constant. The temperature at the shock wave's position is expressed as

$$
T_{\mathrm{e}}=\frac{T_{0}}{1+\frac{\gamma-1}{2} M_{\mathrm{e}}^{2}}
$$

where $T_{0}$ is the temperature at the stagnation point. $T_{0}$ is constant even if the flow conditions change.

\section{Results}

The shock wave's position and the flow velocity were calculated using the method described above for BTF test structures. BTF tests were performed with a synthetic circuit which was composed with a current source supplying interrupting current and a voltage source applying TRV. The test conditions for the interrupting current and TRV were common to all cases. The correlation of these results with the test results was been evaluated.

Table 1 shows four representative test structures. Case $\mathrm{A}$ is a structure whose throat length and divergence angle are 1 (a.u.) and 1 (a.u.), respectively. Including the four test structures shown in Table 1, eleven test structures were built for the interrupting test. The following calculations of the shock wave position and flow velocity are for these seven cases.

In each case, several shots were performed over a range from the expected minimum arcing time to the maximum arcing time.

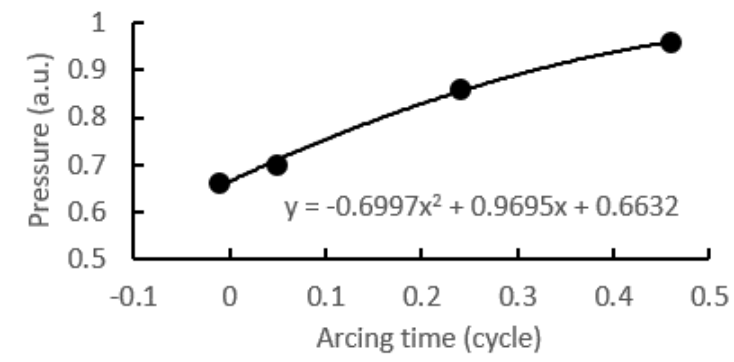

Figure 4. Measured pressure in pressure build-up chamber.

The stagnation pressure $P_{0}$ was set on the basis of the pressures measured with a piezoelectric pressure sensor in the pressure build-up chamber at current zero. The measured pressures of Case A in the pressure build-up chamber at current zero are presented in Figure 4. The horizontal axis in this figure is the arcing time, where the expected minimum arcing time is set to zero. The stagnation pressure was multiplied by 0.9 in consideration of the pressure loss from the chamber to the stagnation point. Assuming that the area of the PTFE surface close to the arc is proportional to the pressure in the chamber, the difference in throat length was determined on the basis of the pressure in Case A. The throat diameter was the same in all cases, as were the stroke characteristics of the electrode. This means that in all cases, the pin electrode was at the same position at a given arcing time.

Figures 5 and 6 show the calculated shock wave positions and flow velocities. The shock wave position was measured from the curved end of the tip of the pin electrode. The open and closed circles in the figures represent interrupting successes and failures, respectively. For the medium arcing times from 0.1 to 0.25 cycle, the cases showing positions at more than $9 \mathrm{~mm}$ were successful. For the short arcing times from around 0 to 0.1 cycle, the cases of more than 0.57 (a.u.) were successful. The predicted shock wave positions for the medium arcing times and predicted flow velocities for the short arcing times correlated well with the test results. However, the calculations for the arcing times longer than 0.25 cycle were not correlated with the test results. It is considered that the volume between the pin electrode and the nozzle was enlarged in the case of the longer arcing times, and this caused local weak points in the dielectric strength. The above criteria might be changing in the rated voltage. Thus, several experiments should be done before applying the method to other rated high-voltage circuit breaker.

Figures 7 (a) and 7 (b) show the position of the shock wave and the flow velocity in calculation, depending on the throat length and the divergence angle for Cases A-D. When the shock wave for a medium arcing time is located downstream, the flow velocity for the case of a short arcing time is small (Case A). In contrast, when the flow velocity for the short arcing 


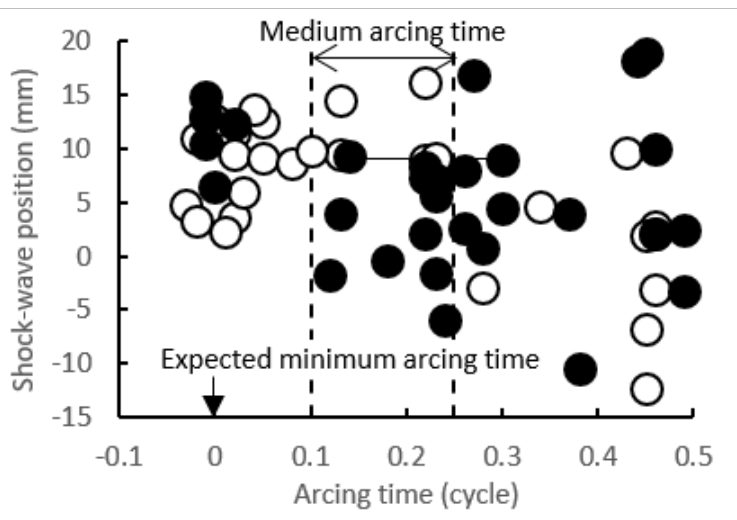

Figure 5. Shock wave position.

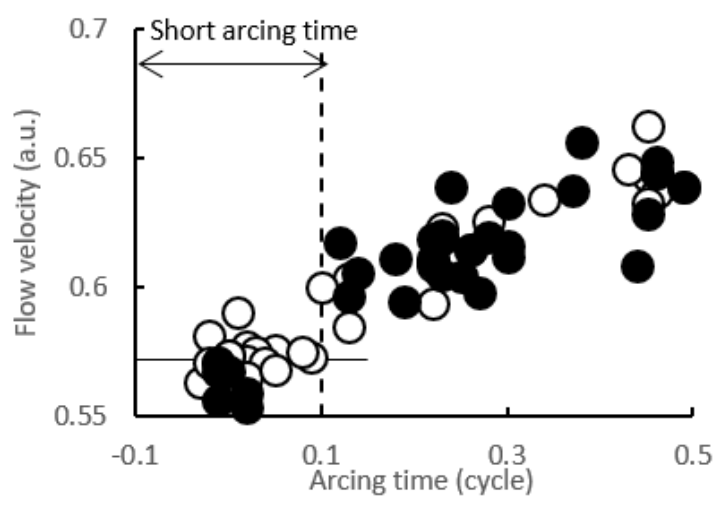

Figure 6. Flow velocity.

time is large, the shock wave for the medium arcing time is located upstream, in front of the tip of the pin electrode (Case B). Since the throat length of Case B is shorter than in Case A, the cross-sectional area of the tip of the pin electrode is larger and the shock wave is generated on the upstream side. As the divergence angle is widened, the flow velocity in the case of the short arcing time increases, whereas the shock wave in the case of the medium arcing time is generated on the upstream side (Case C). Cases B and $\mathrm{C}$, whose shock waves are generated upstream, fail the interrupting test for the medium arcing time. On the other hand, Cases B and C whose flow velocities are large pass the interrupting test for the short arcing time. These results show that the dielectric strength from a short arcing time to a medium arcing time can be evaluated with the method described here. The dielectric strength in long arcing time should be evaluated with two dimensional CFD simulation. In the next step, the dielectric strength is evaluated using with CFD simulation.

\section{Conclusions}

Performance indexes evaluating the dielectric strength, i.e., the flow velocity and shock wave position, were introduced for theoretically evaluating the physical properties of supersonic flow in the nozzle in reference with interrupting test results. It was found that this

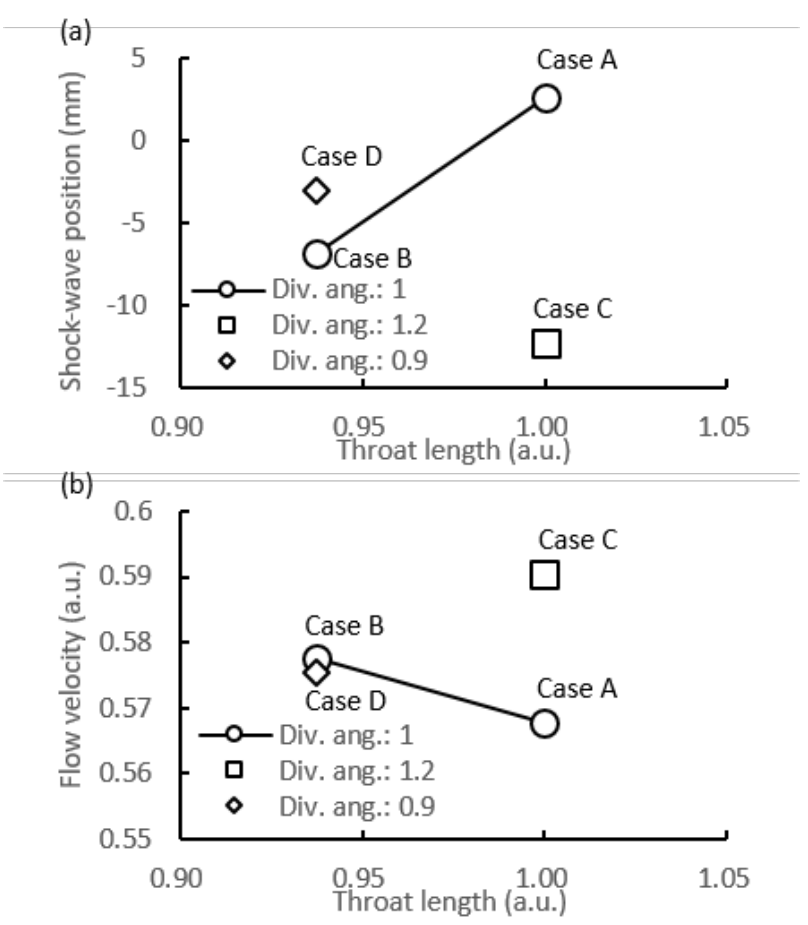

Figure 7. Change in shock wave position (a) and flow velocity (b) depending on throat length and divergence angle.

method can be used to evaluate the dielectric strength in cases ranging from short to medium arcing times.

\section{References}

[1] C. B. Ruchti and L. Niemeyer. Ablation Controlled Arcs. IEEE Trans. Plasma Sci., 14:423-434, 1986. doi:10.1109/TPS. 1986.4316570 .

[2] P. Chévrier et al. Industrial applications of high-, medium- and low-voltage arc modeling. J. Phys. D: Appl. Phys., 30:1346-1355, 1997. doi:10.1088/0022-3727/30/9/010.

[3] M. Seeger et al. An integral arc model for ablation controlled arcs based on cfd simulations. J. Phys. D: Appl. Phys., 39:2180-2191, 2006. doi: 10.1088/0022-3727/39/10/029.

[4] K. Bousoltane et al. Analysis of the breakdown in single-chamber circuit breaker. 18eme Congres Francais de Mecanique Grenoble, 2007.

[5] D. Bešlija et al. Calculation of Steady $\mathrm{SF}_{6}$ Gas Flow through a $420 \mathrm{kV}$ Circuit Breaker Nozzle and Electric Field Distribution. Journal of Energy and Power Engineering, 8:1964-1973, 2014.

[6] K. Y. Park and M. T. C. Fang. Mathematical Modeling of $\mathrm{SF}_{6}$ Puffer Circuit Breakers I: High Current Region. IEEE Trans. on Plasma Science, 24(2):490-502, 1996. doi:10.1109/27.510015.

[7] Y. Yokomizu et al. Dependence of Arc Interrupting Capability on Spatial Distribution of Airflow Velocity in Air-Blast Flat-Type Quenching Chamber. IEEE Trans. on Power Delivery, 18(1):101-106, 2003. doi:10.1109/TPWRD.2002.806687.

[8] H. W. Liepmann and A. Roshko. Elements of Gas Dynamics. Dover Publications, 2002. 